\title{
The Complete Solution of some Kinds of Linear Third Order Partial Differential Equations with Three Independent Variables and variable coefficients
}

Rusul Hassan Naser, Wafaa Hadi Hanoon, Layla Abd Al-Jaleel Mohsin

University of Kufa, Najaf, IRAQ, Department of Computer Science Faculty of Education for Women rusulh.nasir@uokufa.edu.iq

University of Kufa, Najaf, IRAQ, Department of Computer Science Faculty of Education for Women wafaah.hannon@uokufa.edu.iq

University of Kufa, Najaf, IRAQ, Department of Computer Science Faculty of Education for Women Laila.alyasiri@uokufa.edu.iq

\section{ABSTRACT}

In this paper we find the complete solution of some kinds of linear third order partial differential equations of variable coefficients with three independent variables which have the general form

$$
\begin{aligned}
& A Z_{x x x}+B Z_{y y y}+C Z_{t t t}+D Z_{x x y}+E Z_{x x t}+F Z_{x y y}+G Z_{y y t}+H Z_{x t t}+I Z_{y t t}+J Z_{x y t}+ \\
& K Z_{x x}+L Z_{y y}+M Z_{t t}+N Z_{x y}+O Z_{x t}+P Z_{y t}+Q Z_{x}+R Z_{y}+S Z_{t}+T Z=0,
\end{aligned}
$$

Where $A, B, \ldots, T$ are variable coefficients. By use the some assumptions will transform the above equation to the nonlinear second order ordinary differential equations

\section{Indexing terms/Keywords}

linear Partial Differential Equations ; Third Order ; Three Independent Variables

\section{Academic Discipline And Sub-Disciplines}

Applied Mathematics, Differential Equations

\section{SUBJECT CLASSIFICATION}

E.g., Mathematics Subject Classification; Library of Congress Classification

\section{INTRODUCTION}

The differential equations are very importance in the plenty of the fields of the science as Physics, Chemistry and other sciences, therefore plenty of the scientists are studied this subject. Hani [6], and by the assumed

$Z(x, y, t)=e^{\int u(x) d x+\int v(y) d y+\int w(t) d t}$ found the solution to the linear second order partial differential equations with independent variables and of the form

$$
\mathrm{AZ}_{\mathrm{xx}}+\mathrm{BZ}_{\mathrm{xy}}+\mathrm{CZ}_{\mathrm{xt}}+\mathrm{DZ} \mathrm{yy}_{\mathrm{yt}}+\mathrm{EZ}_{\mathrm{tt}}+\mathrm{GZ}_{\mathrm{x}}+\mathrm{HZ}_{\mathrm{y}}+\mathrm{IZ}_{\mathrm{t}}+\mathrm{JZ}=\mathrm{O}
$$

Where $A, B, C, \ldots, I$ and $J$ are arbitrary constants .

Hanoon [7], and by the assumptions

$$
Z(x, y)=\mathrm{e}^{\int \frac{u(x)}{x} d x+\int v(y) d y}, Z(x, y)=\mathrm{e}^{\int u(x) d x+\int \frac{v(y)}{y} d y} \quad \text { and } \quad Z(x, y)=\mathrm{e}^{\int \frac{u(x)}{x} d x+\int \frac{v(y)}{y} d y}
$$

found the solution to the linear second order partial differential equations with coefficients variable and of the form

$$
\mathrm{A}(\mathrm{x}, \mathrm{y}) \mathrm{Z}_{\mathrm{xx}}+\mathrm{B}(\mathrm{x}, \mathrm{y}) \mathrm{Z}_{\mathrm{xy}}+\mathrm{C}(\mathrm{x}, \mathrm{y}) \mathrm{Z}_{\mathrm{yy}}+\mathrm{D}(\mathrm{x}, \mathrm{y}) \mathrm{Z}_{\mathrm{x}}+\mathrm{E}(\mathrm{x}, \mathrm{y}) \mathrm{Z}_{\mathrm{y}}+F(x \cdot y) \mathrm{Z}=0
$$

Where some of $A(x, y), B(x, y), C(x, y), D(x, y), E(x, y)$ and $F(x, y)$ are functions of $x$ or $y$ or both $x$ and $y$.

Dzurina , Thandapani and Tamilvanan [ 5 ], studied the oscillation of solutions to the third-order neutral differential equations

$\left.a(t)\left([x(t) \pm p(t) x(\delta(t))]^{\prime \prime}\right)^{a}\right)^{\prime}+q(t) x^{a}(\tau(t))=0$

Sufficient conditions are established so that every solution is either oscillatory or converges to zero. 
Ketap [8],studied the linear third order partial differential equations, with constant coefficients which have the form $A Z_{x x x}+B Z_{y y y}+C Z_{x x y}+D Z_{x y y}+E Z_{x x}+F Z_{y y}+G Z_{x y}+H Z_{x}+I Z_{y}+J Z=0$,

Where $\mathrm{A}, \ldots \mathrm{I}$ and $\mathrm{J}$ are arbitrary constants , and used the assumption $Z(x, y)=\mathrm{e}^{\int u(x) d x+\int v(y) d y}$ to find the complete solution of it.

Liu, Ume, Anderson and Kang [9], studied singular nonlinear third-order differential equation

Ademola , Ogundiran , Arawomo and Adesina [1], studied boundedness of solutions of the third nonlinear differential equation

$\bar{x}+f(x)+g(\dot{x})+h(x)=p\left(t, x, x_{s} \tilde{x}\right)$, or its equivalent system of differential equations

$\dot{x}=y_{v} \dot{y}=z_{v} z=p(t, x, y, z)-f(z)-g(y)-h(x)$

where $f_{x} g_{x} h \in C\left(R_{x} R\right)$.

$p \in C\left(R^{+} \times R \times R \times R, R\right), R^{+}=[0, \infty)$

and $R=(-\infty, \infty)$

Clarkson, Mansfield and Priestley [3], studies symmetry reductions of a class of nonlinear third-order partial differential equations

$u_{t}-\epsilon u_{x x t}+2 k u_{x}=w u_{x x x}+\alpha w u_{x}+\beta u_{x} u_{x x}$.

where $\epsilon_{,} k, \alpha$ and $\beta$ are arbitrary constants.

This paper is devoted to solve the linear third order partial differential equations with three independent variables of the general form

$$
\begin{aligned}
& A Z_{x x x}+B Z_{y y y}+C Z_{t t t}+D Z_{x x y}+E Z_{x x t}+F Z_{x y y}+G Z_{y y t}+H Z_{x t t}+I Z_{y t t}+J Z_{x y t}+ \\
& K Z_{x x}+L Z_{y y}+M Z_{t t}+N Z_{x y}+O Z_{x t}+P Z_{y t}+Q Z_{x}+R Z_{y}+S Z_{t}+T Z=0,
\end{aligned}
$$

where $A, B, \ldots, T$ are variable coefficients.

By using the assumptions

$$
\begin{aligned}
& Z(x, y, t)=e^{\int \frac{u(x)}{x} d x+\int \frac{v(y)}{y} d y+\int \frac{w(t)}{t} d t}, Z(x, y, t)=e^{\int \frac{u(x)}{x} d x+\int \frac{v(y)}{y} d y+\int w(t) d t} \\
& , Z(x, y, t)=e^{\int \frac{u(x)}{x} d x+\int v(y) d y+\int \frac{w(t)}{t} d t} \text { and } Z(x, y, t)=e^{\int u(x) d x+\int v(y) d y+\int \frac{w(t)}{t} d t}
\end{aligned}
$$

\section{The Complete Solution of linear Third Order partial Differential Equations with Three independent variables}

My aim in this section is to solve the linear third order of partial differential equations, with three independent variables which have the general form

$$
\begin{aligned}
& A Z_{x x x}+B Z_{y y y}+C Z_{t t t}+D Z_{x x y}+E Z_{x x t}+F Z_{x y y}+G Z_{y y t}+H Z_{x t t}+I Z_{y t t}+J Z_{x y t}+ \\
& K Z_{x x}+L Z_{y y}+M Z_{t t}+N Z_{x y}+O Z_{x t}+P Z_{y t}+Q Z_{x}+R Z_{y}+S Z_{t}+T Z=0,
\end{aligned}
$$

where $A, B, \ldots, T$ are variable coefficients.

So, for this purpose we will search functions $u(x), v(y)$ and $w(t)$ such that the assumptions

$$
\begin{aligned}
& Z(x, y, t)=e^{\int \frac{u(x)}{x} d x+\int \frac{v(y)}{y} d y+\int \frac{w(t)}{t} d t}, Z(x, y, t)=e^{\int \frac{u(x)}{x} d x+\int \frac{v(y)}{y} d y+\int w(t) d t}, \\
& Z(x, y, t)=e^{\int \frac{u(x)}{x} d x+\int v(y) d y+\int \frac{w(t)}{t} d t} \text { and } Z(x, y, t)=e^{\int u(x) d x+\int v(y) d y+\int \frac{w(t)}{t} d t}
\end{aligned}
$$


To give the complete solution to the above equation we need to consider many kinds :

\section{Kind (1):}

$\mathrm{A}_{1} t^{2} y \mathrm{Z}_{y t t}+\mathrm{A}_{2} t x y \mathrm{Z}_{x y t}+\mathrm{A}_{3} x^{2} \mathrm{Z}_{x x}+A_{4} x t \mathrm{Z}_{x t}=0$.

\section{Kind (2):}

$\mathrm{A}_{1} \mathrm{Z}_{\mathrm{ttt}}+\mathrm{A}_{2} x^{2} \mathrm{Z}_{x x t}+\mathrm{A}_{3} y \mathrm{Z}_{y t t}+\mathrm{A}_{4} y^{2} \mathrm{Z}_{y y t}+\mathrm{A}_{5} x \mathrm{Z}_{x}=0$.

\section{Kind (3):}

$\mathrm{A}_{1 \text { yyy }} \mathrm{Z}_{2}+\mathrm{A}_{x y y}+\mathrm{A}_{3} t^{2} \mathrm{Z}_{t t}+\mathrm{A}_{4} x \mathrm{Z}_{x y}=0$

\section{Kind (4):}

$\mathrm{A}_{1} Z_{x x y}+\mathrm{A}_{2} t^{2} Z_{t t x}+\mathrm{A}_{3} Z_{x y}=0$

Where $\mathrm{A}_{1} \mathrm{~A}_{2}, \mathrm{~A}_{3}, \mathrm{~A}_{4}$ and $\mathrm{A}_{5}$ are real constants.

kind (1): By using the assumption

$Z=e^{\int \frac{u(x)}{x} d x+\int \frac{v(y)}{y} d y+\int \frac{w(t)}{t} d t}$, the equation $\mathrm{A}_{1} t^{2} y Z_{y t t}+\mathrm{A}_{2} t x y Z_{x y t}+\mathrm{A}_{3} x^{2} Z_{x x}+A_{4} x t Z_{x t}=0$

Transforms to the form

$A_{1}\left(t w^{\prime}(t)+w^{2}(t)-w(t)\right) v(y)+A_{2}(u(x) v(y) w(t))+A_{3}\left(x u^{\prime}(x)+u^{2}(x)-u(x)\right)+A_{4}(u(x) w(t))=0$

This equation can't separate the variables, so we suppose that $w(t)=\lambda_{1}$, where $\lambda_{1}$ is arbitrary constants, then

$A_{1}\left(\lambda_{1}^{2}-\lambda_{1}\right) v(y)+A_{2} \lambda_{1} u(x) v(y)+A_{3}\left(x u^{\prime}(x)+u^{2}(x)-u(x)\right)+A_{4} \lambda_{1} u(x)=0$

$v(y)=\frac{-A_{3}\left(x u^{\prime}(x)+u^{2}(x)-u(x)\right)-A_{4} \lambda_{1} u(x)}{A_{1}\left(\lambda_{1}^{2}-\lambda_{1}\right)+A_{2} \lambda_{1} u(x)}=-\lambda_{2}^{2} \Rightarrow v(y)=-\lambda_{2}^{2}$

$A_{3}\left(x u^{\prime}(x)+u^{2}(x)-u(x)\right)+A_{4} \lambda_{1} u(x)-A_{1} \lambda_{2}^{2}\left(\lambda_{1}^{2}-\lambda_{1}\right)-A_{2} \lambda_{2}^{2} \lambda_{1} u(x)=0$

$x u^{\prime}(x)+u^{2}(x)+\left(\frac{A_{4} \lambda_{1}-A_{2} \lambda_{2}^{2} \lambda_{1}-A_{3}}{A_{3}}\right) u(x)-\frac{A_{1} \lambda_{2}^{2}\left(\lambda_{1}^{2}-\lambda_{1}\right)}{A_{3}}=0$

$x u^{\prime}(x)+u^{2}(x)+B_{1} u(x)+B_{2}=0$

$; B_{1}=\frac{A_{4} \lambda_{1}-A_{2} \lambda_{2}^{2} \lambda_{1}-A_{3}}{A_{3}}$ and $B_{2}=\frac{A_{1} \lambda_{2}^{2}\left(\lambda_{1}^{2}-\lambda_{1}\right)}{A_{3}}$

$x u^{\prime}(x)+\left(u(x)+\frac{B_{1}}{2}\right)^{2}+B_{2}-\frac{B_{1}^{2}}{4}=0$

i) If $B_{2} \neq \frac{B_{1}^{2}}{4}$ we get $\int \frac{\mathrm{du}}{\left(u(x)+\frac{\mathrm{B}_{1}}{2}\right)^{2}+\mathrm{f}_{1}^{2}}+\int \frac{\mathrm{dx}}{\mathrm{x}}=\int 0 ; \mathrm{f}_{1}^{2}=\mathrm{B}_{2}-\frac{\mathrm{B}_{1}^{2}}{4}$ 
$\frac{1}{\mathrm{f}_{1}} \tan ^{-1}\left(\frac{\mathrm{u}(\mathrm{x})+\frac{\mathrm{B}_{1}}{2}}{\mathrm{f}_{1}}\right)=-\ln (c x) ; c x>0 \Rightarrow \mathrm{u}(x)=-\mathrm{f}_{1} \tan \left(\mathrm{f}_{1} \ln (c x)\right)-\frac{B_{1}}{2}$

then the complete solution of equation (1), is given by:

$$
\mathrm{Z}(x, y, t)=\mathrm{e}^{\int \frac{-\mathrm{f}_{1} \tan \left(\mathrm{f}_{1} \ln (c x)\right)-\frac{B_{1}}{2}}{x}-j \frac{\lambda_{2}^{2}}{y} d y+\int \frac{\lambda_{1}}{t} d t}
$$

$$
=\mathrm{e}^{\ln \left(\cos \left(f_{1} \ln (c x)\right)\right)-\frac{B_{1}}{2} \ln x-\lambda_{2}^{2} \ln y+\lambda_{1} \ln t+c_{1}} ; c x>0, y>0, t>0
$$

$Z(x, y, t)=k x \quad \frac{-A_{4} \lambda_{1}+A_{2} \lambda_{2}^{2} \lambda_{1}+A_{3}}{2 A_{3}} y^{-\lambda_{2}^{2}}{ }_{t} \lambda_{1} \cos \left(f_{1} \ln (c x)\right) ; f_{1}=\sqrt{\frac{4 A_{1} A_{3} \lambda_{2}^{2}\left(\lambda_{1}^{2}-\lambda_{1}\right)-\left(A_{4} \lambda_{1}-A_{2} \lambda_{2}^{2} \lambda_{1}-A_{3}\right)^{2}}{4 A_{3}^{2}}}$,

$k=e^{c_{1}}, c x>0$

Where $\mathrm{K}, \mathrm{c}, \lambda_{1}$ and $\lambda_{2}$ are arbitrary constants.

Domain : $\quad \mathrm{x}>0,-\infty<\mathrm{y}<\infty,-\infty<\mathrm{t}<\infty$.

ii) If $B_{2}=\frac{B_{1}^{2}}{4}$ we get $\Rightarrow \int \frac{d u}{\left(u+\frac{B_{1}}{2}\right)^{2}}+\int \frac{d x}{x}=\int 0 \Rightarrow u(x)=\frac{1}{\ln c x}-\frac{B_{1}}{2} ; c x>0$

then the complete solution of equation (1), is given by:

$Z(x, y, t)=e^{\int \frac{\left(\frac{1}{\ln c x}-\frac{B_{1}}{2}\right)}{x} d x+\int \frac{-\lambda_{2}^{2}}{y} d y+\int \frac{\lambda_{1}}{t} d t}$

$Z(x, y, t)=k y^{-\lambda_{2}^{2}} t^{\lambda_{1}} x \underbrace{\frac{-A_{4} \lambda_{1}+A_{2} \lambda_{2}^{2} \lambda_{1}+A_{3}}{2 A_{3}}} \ln c x ; k=e^{c_{1}}, c x>0$

Where $\mathrm{K}, \mathrm{c}, \lambda_{1}$ and $\lambda_{2}$ are arbitrary constants.

Domain : $\quad x>0,-\infty<y<\infty,-\infty<t<\infty$

kind (2) : By using the assumption

$Z(x, y, t)=e^{\int \frac{u(x)}{x} d x+\int \frac{v(y)}{y} d y+\int w(t) d t}$, the equation $\mathrm{A}_{1} \mathrm{Z}_{\mathrm{ttt}}+\mathrm{A}_{2} x^{2} \mathrm{Z}_{x x t}+\mathrm{A}_{3} y \mathrm{Z}_{y t t}+\mathrm{A}_{4} y^{2} \mathrm{Z}_{y y t}+\mathrm{A}_{5} x Z_{x}=0$

Transforms to the form

$\mathrm{A}_{1}\left(w^{\prime \prime}(t)+3 w(t) w^{\prime}(t)+w^{3}(t)\right)+\mathrm{A}_{2}\left(x u^{\prime}(x)+u^{2}(x)-u(x)\right) w(t)+\mathrm{A}_{3}\left(w^{\prime}(t)+w^{2}(t)\right) v(y)+$

$\mathrm{A}_{4}\left(y v^{\prime}(y)+v^{2}(y)-v(y)\right) w(t)+\mathrm{A}_{5} u(x)=0$

This equation can't separate the variables, so we suppose that $w(t)=\lambda_{1} \quad$, where $\lambda_{1}$ is arbitrary constants, then 
$\Rightarrow \mathrm{A}_{1} \lambda_{1}^{3}+\mathrm{A}_{2} \lambda_{1}\left(x u^{\prime}(x)+u^{2}(x)-u(x)\right)+\mathrm{A}_{3} \lambda_{1}^{2} v(y)+\mathrm{A}_{4} \lambda_{1}\left(y v^{\prime}(y)+v^{2}(y)-v(y)\right)+\mathrm{A}_{5} u(x)=0$

This equation is variable separable equation [4].

$\Rightarrow A_{2} \lambda_{1}\left(x u^{\prime}(x)+u^{2}(x)-u(x)\right)+A_{5} u(x)=-A_{4} \lambda_{1}\left(y v^{\prime}(y)+v^{2}(y)-v(y)\right)-A_{3} \lambda_{1}^{2} v(y)-A_{1} \lambda_{1}^{3}=-\lambda_{2}$

$\Rightarrow x u^{\prime}(x)+u^{2}(x)+\left(\frac{A_{5}}{A_{2} \lambda_{1}}-1\right) u(x)+\frac{\lambda_{2}}{A_{2} \lambda_{1}}=0$

Let $\mathrm{B}_{1}=\frac{A_{5}}{A_{2} \lambda_{1}}-1$ and $\mathrm{B}_{2}=\frac{\lambda_{2}}{A_{2} \lambda_{1}} \Rightarrow x u^{\prime}(x)+u^{2}(x)+\mathrm{B}_{1} u(x)+\mathrm{B}_{2}=0$

$y v^{\prime}(y)+v^{2}(y)+\left(\frac{A_{3} \lambda_{1}^{2}}{A_{4} \lambda_{1}}-1\right) v(y)+\frac{A_{1} \lambda_{1}^{3}-\lambda_{2}}{A_{4} \lambda_{1}}=0$

Let $\mathrm{B}_{3}=\frac{A_{3} \lambda_{1}^{2}}{A_{4} \lambda_{1}}-1$ and $\mathrm{B}_{4}=\frac{A_{1} \lambda_{1}^{3}-\lambda_{2}}{A_{4} \lambda_{1}} \Rightarrow y v^{\prime}(y)+v^{2}(y)+\mathrm{B}_{3} v(y)+\mathrm{B}_{4}=0$

The equation (4), is similar to equation (2), then the same method the solution of it is given by :

i) If $\quad B_{2} \neq \frac{B_{1}^{2}}{4}$; we get $\Rightarrow \mathrm{u}(x)=-\mathrm{f}_{1} \tan \left(\mathrm{f}_{1} \ln (c x)\right)-\frac{B_{1}}{2} ; \mathrm{f}_{1}^{2}=\mathrm{B}_{2}-\frac{\mathrm{B}_{1}^{2}}{4}$

ii) If $B_{2}=\frac{B_{1}^{2}}{4}$ we get $\Rightarrow u(x)=\frac{1}{\ln c x}-\frac{B_{1}}{2} ; c x>0$

Now the equation(5), is similar to equation (2), then the same method the solution of it is given by :

i) If $\quad B_{4} \neq \frac{B_{3}}{4}$; we get $\Rightarrow \mathrm{v}(y)=-\mathrm{f}_{2} \tan \left(\mathrm{f}_{2} \ln \left(c_{1} y\right)\right)-\frac{B_{3}}{2} \quad ; \mathrm{f}_{2}^{2}=\mathrm{B}_{4}-\frac{\mathrm{B}_{3}^{2}}{4} \quad, \quad \mathrm{c}_{1} y>0$

ii) If $B_{4}=\frac{B_{3}}{4}$ we get $\Rightarrow v(y)=\frac{1}{\ln c_{1} y}-\frac{B_{3}}{2} \quad ; c_{1} y>0$

Then the complete solution of the equation (3), is given by :

i) If $B_{2} \neq \frac{B_{1}^{2}}{4}$ and $B_{4} \neq \frac{B_{3}}{4}$, we get

$\Rightarrow Z(x, y, t)=e^{\int \frac{-\mathrm{f}_{1} \tan \left(\mathrm{f}_{1} \ln (c x)\right)-\frac{B_{1}}{2}}{x} d x} \times \mathrm{e}^{\int \frac{\mathrm{f}_{2} \tan \left(\mathrm{f}_{2} \ln \left(c_{1} y\right)\right)-\frac{B_{3}}{2}}{y}} d y+\int \lambda_{1} \mathrm{dt}$

$Z(x, y, t)=\mathrm{K} x \mathrm{C}^{-\frac{B_{1}}{2}} e^{-\frac{B_{3}}{2}} e^{\lambda_{1} t} \cos \left(\mathrm{f}_{1} \ln (c x)\right) \cos \left(\mathrm{f}_{2} \ln \left(c_{1} y\right)\right) ; \mathrm{K}=\mathrm{e}^{\mathrm{c}}{ }^{2}, \mathrm{~B}_{1}=\frac{A_{5}}{A_{2} \lambda_{1}}-1, \mathrm{~B}_{3}=\frac{A_{3} \lambda_{1}^{2}}{A_{4} \lambda_{1}}-1$

$\mathrm{f}_{1}=\sqrt{\frac{\lambda_{2}}{A_{2} \lambda_{1}}-\left(\frac{A_{5}}{2 A_{2} \lambda_{1}}-\frac{1}{2}\right)^{2}}, \mathrm{cx}>0, \mathrm{c}_{1} y>0$ and $\mathrm{f}_{2}=\sqrt{\frac{A_{1} \lambda_{1}^{3}-\lambda_{2}}{A_{4} \lambda_{1}}-\frac{\left(A_{3} \lambda_{1}^{2}-A_{4} \lambda_{1}\right)^{2}}{4 A_{4}^{2} \lambda_{1}^{2}}}$ 
Where $\mathrm{K}, \mathrm{c}, \mathrm{c} 1, \lambda_{1}$ and $\lambda_{2}$ are arbitrary constants.

Domain : $\mathrm{x}>0, \mathrm{y}>0,-\infty<\mathrm{t}<\infty$

ii) If $B_{2} \neq \frac{B_{1}^{2}}{4}$ and $B_{4}=\frac{B_{3}}{4}$, we get

$Z(x, y, t)=\mathrm{K} x{ }^{-\frac{B}{2}} y^{2} e^{\lambda_{1} t} \cos \left(\mathrm{f}_{1} \ln (c x)\right) \ln \left(c_{1} y\right) ; \mathrm{K}=\mathrm{e}^{\mathrm{c}_{2}}, \mathrm{~B}_{1}=\frac{A_{5}}{A_{2} \lambda_{1}}-1 \quad, \mathrm{~B}_{3}=\frac{A_{3} \lambda_{1}^{2}}{A_{4} \lambda_{1}}-1$

$, \mathrm{f}_{1}=\sqrt{\frac{\lambda_{2}}{A_{2} \lambda_{1}}-\left(\frac{A_{5}}{2 A_{2} \lambda_{1}}-\frac{1}{2}\right)^{2}} \quad, \quad \mathrm{cx}>0$ and $\mathrm{c}_{1} y>0$

Where $\mathrm{K}, \mathrm{c}, \mathrm{c} 1, \lambda_{1}$ and $\lambda_{2}$ are arbitrary constants.

Domain: $\mathrm{x}>0, \mathrm{y}>0,-\infty<\mathrm{t}<\infty$

$Z(x, y, t)=e^{\int \frac{1}{\frac{\ln c x}{x}-\frac{B_{1}}{2}} d x+\int \frac{-\mathrm{f}_{2} \tan \left(\mathrm{f}_{2} \ln \left(c_{1} y\right)\right)-\frac{B_{3}}{2}}{y}} d y+\int \lambda_{1} \mathrm{dt}$

$Z(x, y, t)=\mathrm{K} x^{-\frac{B}{2}} y^{-\frac{B}{2}} e^{\lambda_{1} t} \ln (c x) \cos \left(\mathrm{f}_{2} \ln \left(c_{1} y\right)\right) ; \mathrm{K}=\mathrm{e}^{\mathrm{c}}{ }^{2}, \mathrm{~B}_{1}=\frac{A_{5}}{A_{2} \lambda_{1}}-1, \mathrm{~B}_{3}=\frac{A_{3} \lambda_{1}^{2}}{A_{4} \lambda_{1}}-1$

iii) If $B_{2}=\frac{B_{1}^{2}}{4}$ and $B_{4} \neq \frac{B_{3}}{4}$, we get

$, \mathrm{f}_{2}=\sqrt{\frac{A_{1} \lambda_{1}^{3}+A_{3} \lambda_{1}^{2}-\lambda_{2}}{A_{4} \lambda_{1}}-\frac{1}{4}}, \mathrm{cx}>0, \mathrm{c}_{1} y>0$ Where $\mathrm{K}, \mathrm{c}, \mathrm{c} 1, \lambda_{1}$ and $\lambda_{2}$ are arbitrary constants.

Domain: $\mathrm{x}>0, \mathrm{y}>0,-\infty<\mathrm{t}<\infty$

iv) If $B_{2}=\frac{B_{1}^{2}}{4}$ and $B_{4}=\frac{B_{3}}{4}$, we get

$Z(x, y, t)=e^{\int \frac{1}{\ln c x}-\frac{B_{1}}{2}} d x+\int \frac{\frac{1}{\ln c_{1} y}-\frac{B_{3}}{2}}{y} d y+\int \lambda_{1} \mathrm{dt}$

$Z(x, y, t)=\mathrm{K} x \mathrm{P}^{-\frac{B_{1}}{2}} y^{-\frac{B_{3}}{2}} e^{\lambda_{1} t} \ln (c x) \ln \left(c_{1} y\right) ; \mathrm{K}=\mathrm{e}^{\mathrm{c}_{2}}, \mathrm{~B}_{1}=\frac{A_{5}}{A_{2} \lambda_{1}}-1 \quad, \mathrm{~B}_{3}=\frac{A_{3} \lambda_{1}^{2}}{A_{4} \lambda_{1}}-1, \mathrm{cx}>0, \mathrm{c}_{1} y>0$

Where $\mathrm{K}, \mathrm{C}, \mathrm{c} 1$ and $\lambda 1$ are arbitrary constants.

Domain: $\mathrm{x}>0, \mathrm{y}>0,-\infty<\mathrm{t}<\infty$

kind (3) : By using the assumption $Z=e^{\int \frac{u(x)}{x} d x+\int v(y) d y+\int \frac{w(t)}{t} d t}$, the equation

$\mathrm{A}_{1 \text { yyy }}+\mathrm{A}_{2} x \mathrm{Z}_{x y y}+\mathrm{A}_{3} t^{2} \mathrm{Z}_{t t}+\mathrm{A}_{4} x \mathrm{Z}_{x y}=0$ 
Transforms to the form

$\mathrm{A}_{1}\left(v^{\prime \prime}(y)+3 v(y) v^{\prime}(y)+v^{3}(y)\right)+\mathrm{A}_{2} u(x)\left(v^{\prime}(y)+v^{2}(y)\right)+\mathrm{A}_{3}\left(t w^{\prime}(t)+w^{2}(t)-w(t)\right)+$

$\mathrm{A}_{4} u(x) v(y)=0$

This equation can't separate the variables, so we suppose that $v(y)=\lambda_{1} \quad$, where $\lambda_{1}$ is arbitrary constants , then

$\mathrm{A}_{1} \lambda_{1}^{3}+\mathrm{A}_{2} \lambda_{1}^{2} u(x)+\mathrm{A}_{3}\left(t w^{\prime}(t)+w^{2}(t)-w(t)\right)+\mathrm{A}_{4} \lambda_{1} u(x)=0$

This equation is variable separable equation [4].

$\mathrm{A}_{1} \lambda_{1}^{3}+\mathrm{A}_{2} \lambda_{1}^{2} u(x)+\mathrm{A}_{4} \lambda_{1} u(x)=-\mathrm{A}_{3}\left(t w^{\prime}(t)+w^{2}(t)-w(t)\right)=-\lambda_{2}$

$\mathrm{A}_{1} \lambda_{1}^{3}+\mathrm{A}_{2} \lambda_{1}^{2} u(x)+\mathrm{A}_{4} \lambda_{1} u(x)+\lambda_{2}=0 \Rightarrow u(x)=\frac{-\left(\mathrm{A}_{1} \lambda_{1}^{3}+\lambda_{2}\right)}{\mathrm{A}_{2} \lambda_{1}^{2}+\mathrm{A}_{4} \lambda_{1}}$

And $t w^{\prime}(t)+w^{2}(t)-w(t)-\frac{\lambda_{2}}{\mathrm{~A}_{3}}=0$

Let $\mathrm{B}=\frac{\lambda_{2}}{\mathrm{~A}_{3}} \Rightarrow t w^{\prime}(t)+w^{2}(t)-w(t)-B=0$

This equation is variable separable equation [4], we can solve it as follows :

$$
-\frac{\mathrm{dw}}{\mathrm{d}^{2}-\left(w(t)-\frac{1}{2}\right)^{2}}+\frac{\mathrm{dt}}{\mathrm{t}}=0 \quad ; \mathrm{d}^{2}=\mathrm{B}+\frac{1}{4}
$$

i) if $\mathrm{B} \neq-\frac{1}{4}$, we get $\Rightarrow-\frac{1}{\mathrm{~d}} \tanh ^{-1}\left(\frac{\mathrm{w}(\mathrm{t})-\frac{1}{2}}{\mathrm{~d}}\right)=-\ln (c t) \Rightarrow \mathrm{w}(t)=\mathrm{d} \tanh (\mathrm{d} \ln (c t))+\frac{1}{2} ; c t>0$

ii) if $\mathrm{B}=-\frac{1}{4}$, we get $\Rightarrow \frac{\mathrm{dw}}{\left(w(t)-\frac{1}{2}\right)^{2}}+\frac{\mathrm{dt}}{\mathrm{t}}=0 \Rightarrow \mathrm{w}(t)=\frac{1}{\ln (c t)}+\frac{1}{2} \quad ; c t>0$

Then the complete solution of the equation (6), is given by :

i) if $\mathrm{B} \neq-\frac{1}{4}$, we get

$Z(x, y, t)=e^{\int \frac{\frac{-\left(\mathrm{A}_{1} \lambda_{1}^{3}+\lambda_{2}\right)}{\mathrm{A}_{2} \lambda_{1}^{2}+\mathrm{A}_{4} \lambda_{1}}}{x}} d x+\int \lambda_{1} d y+\int \frac{\mathrm{d} \tanh (\mathrm{d} \ln (c t))+\frac{1}{2}}{\mathrm{t}} \mathrm{dt}$

$Z(x, y, t)=\mathrm{K} x^{\frac{-\left(\mathrm{A}_{1} \lambda_{1}^{3}+\lambda_{2}\right)}{\mathrm{A}_{2} \lambda_{1}^{2}+\mathrm{A}_{4} \lambda_{1}}} e^{\lambda_{1}^{y}} t^{\frac{1}{2}} \cosh \left(\left(\sqrt{\frac{\lambda_{2}}{\mathrm{~A}_{3}}+\frac{1}{4}}\right) \ln (c t)\right) \quad ; \mathrm{K}=\mathrm{e}^{\mathrm{c}_{1}}, c t>0$ 
Where $\mathrm{K}, \mathrm{c}, \lambda_{1}$ and $\lambda_{2}$ are arbitrary constants.

Domain : $-\infty<\mathrm{x}<\infty,-\infty<\mathrm{y}<\infty, \mathrm{t}>0$.

ii) if $\mathrm{B}=-\frac{1}{4}$, we get $\Rightarrow Z(x, y, t)=e^{\int \frac{-\left(\mathrm{A}_{1} \lambda_{1}^{3}+\lambda_{2}\right)}{\mathrm{A}_{2} \lambda_{1}^{2}+\mathrm{A}_{4} \lambda_{1}}} e^{x} d x+\int \lambda_{1} d y+\int \frac{\frac{1}{\ln (c t)}+\frac{1}{2}}{\mathrm{t}} \mathrm{dt}$

$Z(x, y, t)=\mathrm{K} x^{\frac{-\left(\mathrm{A}_{1} \lambda_{1}^{3}+\lambda_{2}\right)}{\mathrm{A}_{2} \lambda_{1}^{2}+\mathrm{A}_{4} \lambda_{1}}} e^{\lambda_{1}{ }^{y} t^{\frac{1}{2}}} \ln (c t) \quad ; \mathrm{K}=\mathrm{e}^{\mathrm{c}_{1}}, c t>0$

Where $\mathrm{K}, \mathrm{c}, \lambda_{1}$ and $\lambda_{2}$ are arbitrary constants.

Domain : $-\infty<x<\infty,-\infty<y<\infty, t>0$.

kind (4): By using the assumption

$Z(x, y, t)=e^{\int u(x) d x+\int v(y) d y+\int \frac{w(t)}{t} d t}$, the equation $\mathrm{A}_{1} Z_{x x y}+\mathrm{A}_{2} t^{2} Z_{t t x}+\mathrm{A}_{3} Z_{x y}=0$

Transforms to the form

$\mathrm{A}_{1}\left(u^{\prime}(x)+u^{2}(x)\right) v(y)+\mathrm{A}_{2}\left(t w^{\prime}(t)+w^{2}(t)-w(t)\right) u(x)+\mathrm{A}_{3} u(x) v(y)=0$

This equation can't separate the variables, so we suppose that $w(t)=\lambda_{1} \quad$, where $\lambda_{1}$ is arbitrary constants , then $\mathrm{A}_{1}\left(u^{\prime}(x)+u^{2}(x)\right) v(y)+\mathrm{A}_{2}\left(\lambda_{1}^{2}-\lambda_{1}\right) u(x)+\mathrm{A}_{3} u(x) v(y)=0$

This equation is variable separable equation [4].

$\frac{v(y)}{\mathrm{A}_{2}\left(\lambda_{1}^{2}-\lambda_{1}\right)+\mathrm{A}_{3} v(y)}=-\frac{u(x)}{\mathrm{A}_{1}\left(u^{\prime}(x)+u^{2}(x)\right)}=-\lambda_{2} \Rightarrow v(y)=\frac{\mathrm{A}_{2} \lambda_{2}\left(\lambda_{1}-\lambda_{1}^{2}\right)}{\lambda_{2} \mathrm{~A}_{3}-1}$

And $u^{\prime}(x)+u^{2}(x)-\frac{1}{\mathrm{~A}_{1} \lambda_{2}} u(x)=0$

This equation is similar to Bernoulli equation [2], then the solution of it is given by :

$$
u(x)=\frac{e^{\frac{1}{A_{1} \lambda_{2}} x}}{\int e^{\frac{1}{A_{1} \lambda_{2}} x} d x}
$$

Then the complete solution of the equation (7), is given by:

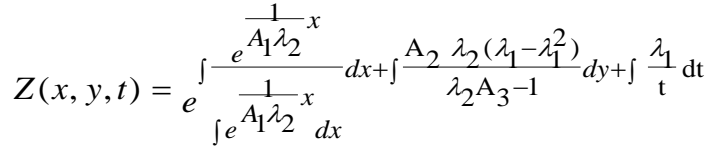

$$
\begin{aligned}
& =e^{\ln \left(\int e^{\frac{1}{A_{1} \lambda_{2}} x} d x+\frac{\mathrm{A}_{2} \lambda_{2}\left(\lambda_{1}-\lambda_{1}^{2}\right)}{\lambda_{2} \mathrm{~A}_{3}-1} y+\lambda_{1} n t\right.} \\
& =\mathrm{K} t^{\lambda_{1}} e^{\frac{1}{A_{1} \lambda_{2}} x+\frac{\mathrm{A}_{2} \lambda_{2}\left(\lambda_{1}-\lambda_{1}^{2}\right)}{\lambda_{2} \mathrm{~A}_{3}-1} y} ; \mathrm{K}=A_{1} \lambda_{2} e^{c}
\end{aligned}
$$


Where $\mathrm{K}, \lambda_{1}$ and $\lambda_{2}$ are arbitrary constant.

Domain : $-\infty<\mathrm{x}<\infty,-\infty<\mathrm{y}<\infty,-\infty<\mathrm{t}<\infty$.

\section{REFERENCES}

1. Ademola T. A. , Ogundiran, M. O., Arawomo , Peter O. and Adesina , O. A.," Boundednes results for a certain third order nonlinear differential equation", Journal of Applied Mathematics and Computation 216 (2010) 30443049.

2. Braun, M., "Differential Equation and Their Applications",4thed. New. York :Spring-verlag, (1993).

3. Clarkson, P.A., Mansfield, E. L. and Priestley, T. J.

" Symmetries of a Class of Nonlinear Third -Order Partial Differential Equations", Journal of Mathl. Cornput. Modelling Vol. 25, No. 8/9, pp. 195-212, (1997).

4. Codington, E.A. ,"An Introduction to Ordinary Differential Equations ",New York: Dover, 1989.

5. Dzurina,J. Thandapani, E. and Tamilvanan, S.

" Oscillation of solutions to third-order half-linear neutral differential equations" , Electron. J. Diff. Equ., Vol. 2012, No. 29, pp. 1-9, (2012).

6. Hani N.N., "On Solutions of Partial Differential Equations of second order with Constant Coefficients", Msc, thesis, University of Kufa,( 2008).

7. Hanoon W.H., "On Solutions of Partial Differential Equations and Their Physical Applications ", Msc, thesis, University of Kufa,, (2009).

8. Ketap, S.N., "The Complete Solution for Some Kinds of Linear Third Order Partial Differential Equations ", Msc, thesis, University of Kufa,( 2011).

9. Liu , Z. ,Ume , J. S., Anderson , D. R .and Kang , S. M. ," Twin monotone positive solutions to a singular nonlinear third-order differential equation" J. Math. Anal. Appl. 334 (2007) 299-313 